\title{
ELECTROCHEMICAL DYEING OF POLYESTER FABRIC USING DISPERSE DYES
}

\author{
K. Kalapriya ${ }^{1, *}$, H. Gurumallesh Prabu ${ }^{2}$ and G. Ramakrishnan ${ }^{3}$ \\ Department of Science and Humanities, Chemistry division, Kumaraguru College of \\ Technology, Coimbatore- 641049, TN, India \\ Department of Industrial Chemistry, Alagappa University, Karaikudi- 630003, TN, India \\ Department of Fashion Technology, Kumaraguru College of Technology, \\ Coimbatore - 641049, TN, India \\ *E-mail: kalapriyaa@gmail.com
}

\begin{abstract}
The electrochemical technique of dyeing assumes greater significance from economical and ecological points of view. An attempt is made with the objectives of (i) dyeing process with the electrical potential application $(2,3,4 \mathrm{~V})$ using DC power supply (ii) enhancing the dyeability by embedding the fabric on graphite electrodes. Cyclic Voltammetric studies and UV-Vis spectral analysis showed that the disperse dyes were electroactive by nature. The dyeability was measured by computer color matching.

Keywords: Electrochemical, Polyester, Disperse, Potential, Dyeing

(C) RASĀYAN. All rights reserved

\section{INTRODUCTION}

Electrochemical dyeing which minimizes the consumption of chemicals is found to be an alternative technique that reduces the dye molecules ${ }^{1,2}$ as it doesn't require any chemical reducing agent. In the direct electrochemical reduction method, reducing agents are replaced by electrons from electric current, and effluent contaminating substances are distributed altogether., ${ }^{3,4}$ Thus, it offers greater economic and environmental benefits in textile processes. ${ }^{5}$ Graphite a very cheap and stable material, seems to be of significant concerning the direct electrochemical reduction. ${ }^{5,6}$ Textile applications on the thermal properties of polyester fiber are being developed. ${ }^{7}$ In this study an attempt is made to find the dyeability of fabric by application of potential and to improve the dyeability of samples.
\end{abstract}

\section{Materials and Methods}

\section{EXPERIMENTAL}

Polyester fabric was purchased from the local market. The fabric was treated for 30mins with dil.HCl to remove the impurities. The \% weight loss of fabric was noted. The fabric was dyed with disperse dyes (Disperse Orange 3, Disperse Orange 13, Disperse Red 11). A material to liquor ratio (MLR 1:30) was maintained to produce $0.5 \%$ shade.

Dye -1

Commercial Name: Tulastran Fast Orange GR

CI Name (Number): Disperse Orange 3 (11005)

CAS Number: $730-40-5$

Chemical name: 4-(4-nitrophenylazo)aniline

Chemical formula: $\mathrm{C}_{12} \mathrm{H}_{10} \mathrm{~N}_{4} \mathrm{O}_{2}$

Rasayan J. Chem., 12(1), 319-323(2019)

http://dx.doi.org/10.31788/RJC.2019.1215004

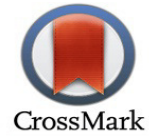


Dye - 2

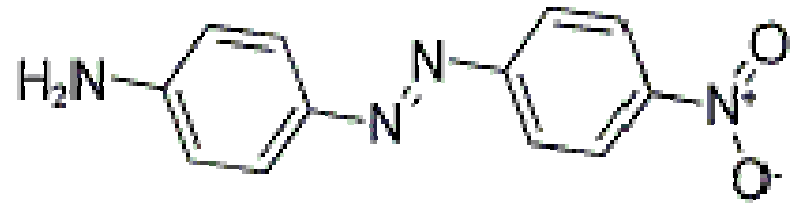

Fig.-1

Commercial Name: Disperse Orange MB

CI Name (Number): Disperse Orange 13 (26080)

CAS Number: 6253-10-7

Chemical name: [4-(Phenylazo)-1-naphthylazo]phenol

Chemical formula: $\mathrm{C}_{22} \mathrm{H}_{16} \mathrm{~N}_{4} \mathrm{O}_{4}$

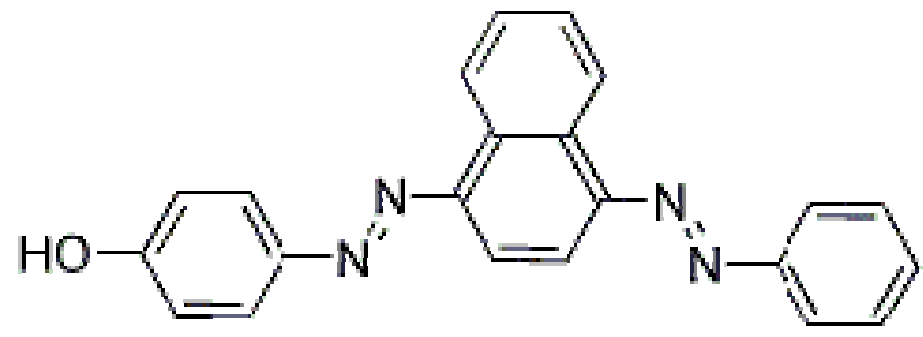

Fig.-2

Dye - 3

Commercial Name: Duratic Red B3B

CI Name (Number): Disperse Red 11 (62015)

CAS Number: 2872-48-2

Chemical name: 1,4-diamino-2-methoxy anthraquinone

Chemical formula: $\mathrm{C}_{15} \mathrm{H}_{12} \mathrm{~N}_{2} \mathrm{O}_{3}$

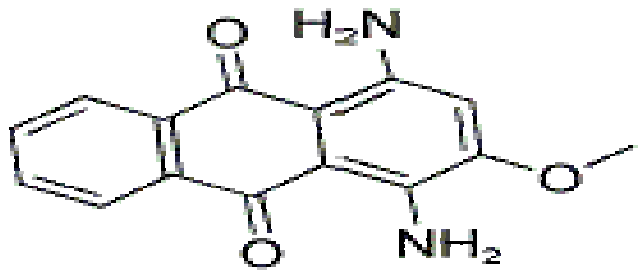

Fig.-3

\section{Dyeing Polyester by Electrochemical Method}

The polyester fabric was immersed in cell construction and other ingredients like dye and auxiliaries (ammonium acetate and acetic acid) were added. Two graphite electrodes were used as anode and cathode. Dyeing was carried for one hour by the application of potential $(2,3$ and $4 \mathrm{~V})$ using a DC power supply unit. Then the dyed samples were taken out and washed well.

\section{UV-Visible Spectral Analysis of Dyes}

\section{RESULTS AND DISCUSSION}

The UV-Vis spectral analysis of the disperse dyes selected was performed with Jasco V-530 UV-Visible spectrophotometer. The $\lambda_{\max }$ of each dye was noted and calibration plots were obtained by measuring the optical density of the dye at different concentrations at respective $\lambda_{\max }$ conditions. The spectral results obtained are tabulated below (Table-1).

Table-1: UV-Visible Spectral Analysis of Dyes

\begin{tabular}{c|c|c|c}
\hline S. No. & $\begin{array}{c}\text { Dye } \\
\text { (Abbreviated as) }\end{array}$ & $\begin{array}{c}\text { Number of Peaks } \\
(\lambda \mathrm{nm})\end{array}$ & $\lambda \max$ \\
\hline 1 & $\begin{array}{c}\text { DISPERSE ORANGE 3 } \\
(\text { DIO3) }\end{array}$ & $\begin{array}{c}3 \\
(430,284,2320\end{array}$ & 430 \\
\hline
\end{tabular}


RASĀYAN J. Chem.

Vol. 12 | No. 1 |319 - 323| January - March | 2019

\begin{tabular}{c|c|c|c}
\hline 2 & $\begin{array}{c}\text { DISPERSE ORANGE 13 } \\
(\text { DIO13) }\end{array}$ & $\begin{array}{c}2 \\
(424,286)\end{array}$ & 424 \\
\hline 3 & $\begin{array}{c}\text { DISPERSE RED 11 } \\
(\text { DIR11) }\end{array}$ & $\begin{array}{c}2 \\
(570,620)\end{array}$ & 620 \\
\hline
\end{tabular}

\section{Cyclic Voltammetric Analysis of Dyes}

The cyclic voltammograms of disperse dyes were performed using CHI-620A workstation with threeelectrode cell assembly (Table-2). The measurement was taken at a scan rate of $0.5 \mathrm{~V} / \mathrm{s}$. Glassy carbon as working, $\mathrm{Ag} / \mathrm{AgCl}$ as a reference, a platinum wire as counter electrodes were used respectively. $0.1 \mathrm{M}$ $\mathrm{KCl}$ was used as supporting electrolyte. Nitrogen gas was used for purging.

Table-2: Cyclic Voltammetric Analysis of Dyes

\begin{tabular}{|c|c|c|c|c|c|c|}
\hline \multirow[t]{2}{*}{ Dye } & \multicolumn{3}{|c|}{ Anodic Peak } & \multicolumn{3}{|c|}{ Cathodic Peak } \\
\hline & Peaks & $\begin{array}{l}\text { Potential } \\
\text { (V) }\end{array}$ & $\begin{array}{l}\text { Current } \\
\text { (A) }\end{array}$ & Peaks & $\begin{array}{l}\text { Potential } \\
\text { (V) }\end{array}$ & $\begin{array}{l}\text { Current } \\
\text { (A) }\end{array}$ \\
\hline $\begin{array}{c}\text { DISPERSE } \\
\text { ORANGE } 3 \\
\text { (DIO3) }\end{array}$ & 1 & 0.1613 & 5.696 e-6 & 1 & -0.7556 & -1.402 e-5 \\
\hline $\begin{array}{c}\text { DISPERSE } \\
\text { ORANGE } 13 \\
\text { (DIO13) }\end{array}$ & - & - & - & 1 & -0.521 & -7.362 e-6 \\
\hline $\begin{array}{c}\text { DISPERSE RED } \\
11 \\
\text { (DIR11) }\end{array}$ & 3 & $\begin{array}{c}0.533 \\
-0.9331 \\
-0.7902\end{array}$ & $\begin{array}{c}4.346 \mathrm{e}-6 \\
-2.950 \mathrm{e}-6 \\
2.354 \mathrm{e}-6\end{array}$ & 2 & $\begin{array}{l}-0.8913 \\
-0.6225\end{array}$ & $\begin{array}{l}-1.031 e-5 \\
-9.978 e-6\end{array}$ \\
\hline
\end{tabular}

From the cyclic voltammetric studies, it was revealed that all dyes showed electro-oxidation and electroreduction reactions and the representative voltammograms are given below (Table-3).

\section{Dyeing of Polyester at 2V}

In the dyeing of polyester with DIO 13 dye, the dyeability and washing fastness of the fabric was moderate and the maximum K/S values of 2.13 for dyed fabric and 1.99 for wash fast fabric samples were obtained (Table-4).

Table-3: UV Spectrum and Cyclic Voltammogram Analysis of Dyes

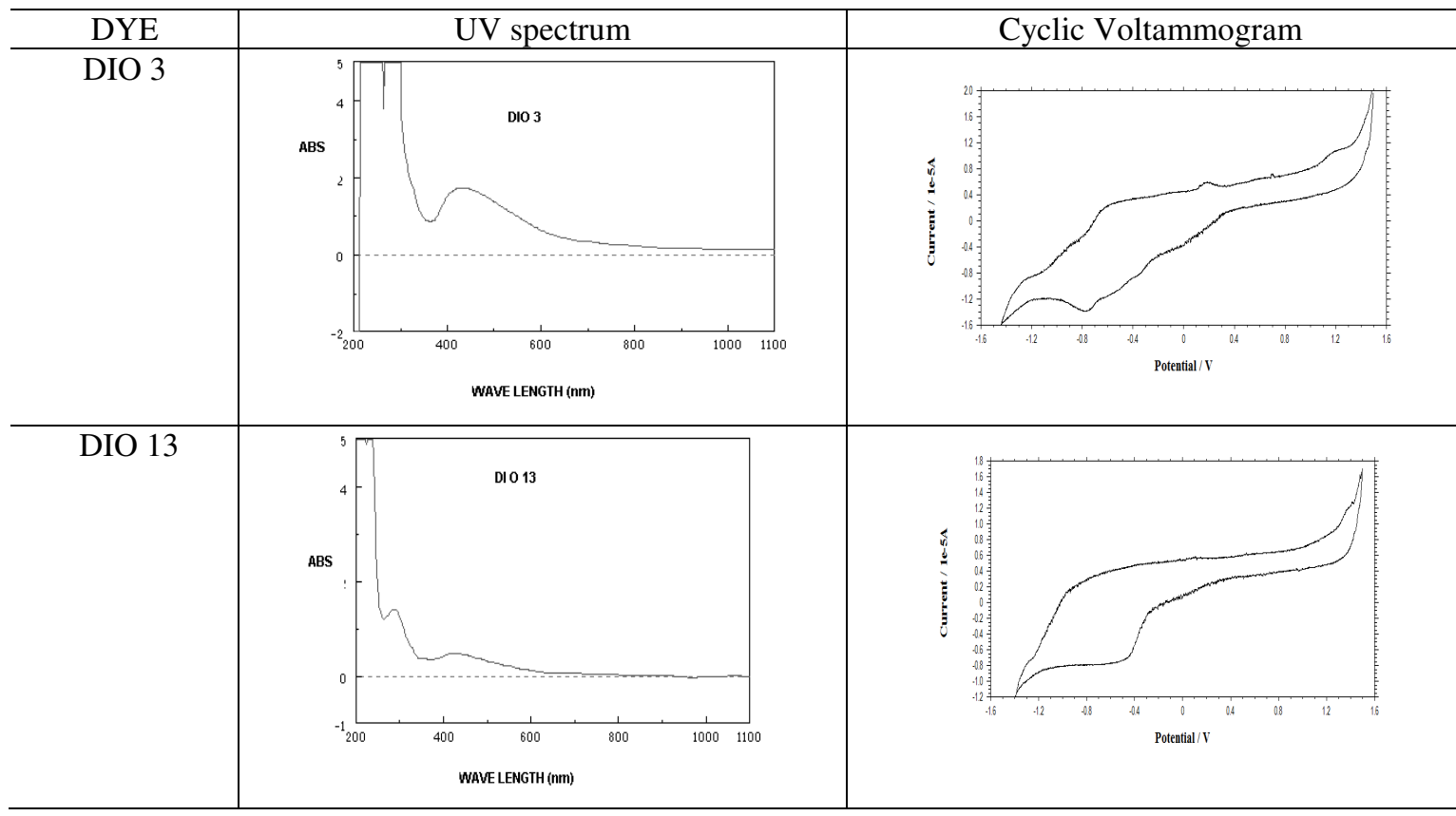


RASĀYAN J. Chem.

Vol. 12 | No. 1 |319 - 323| January - March | 2019

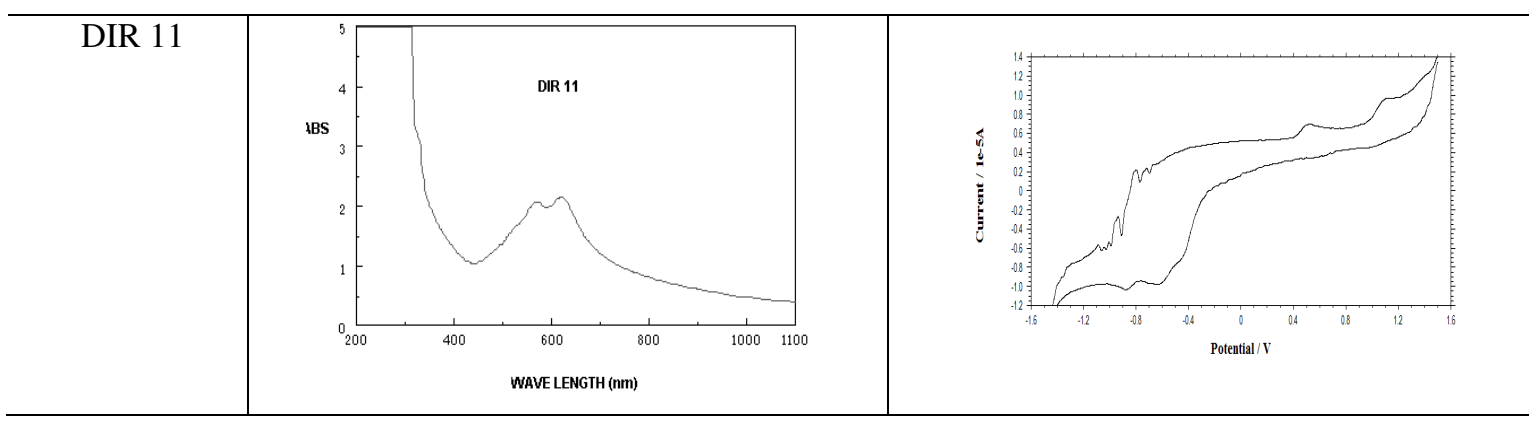

Table-4: Dyeing of Polyester at 2V

\begin{tabular}{c|c|c|c|c|c|c|c|c|c|c|c|c}
\hline \multicolumn{9}{c|}{ CCM Data before Washing } & \multicolumn{6}{c}{ CCM Data after Washing } \\
\hline Dye & $\mathrm{L}^{*}$ & $\mathrm{a}^{*}$ & $\mathrm{~b} *$ & $\mathrm{C}^{*}$ & $\mathrm{H}$ & $\mathrm{K} / \mathrm{S}$ & $\mathrm{L} *$ & $\mathrm{a}^{*}$ & $\mathrm{~b}^{*}$ & $\mathrm{C}^{*}$ & $\mathrm{H}$ & $\mathrm{K} / \mathrm{S}$ \\
\hline DIO 3 & 71.26 & 5.72 & 14.74 & 15.81 & 68.80 & 1.87 & 71.59 & 5.20 & 12.46 & 13.50 & 67.36 & 1.72 \\
\hline DIO 13 & 69.95 & 9.19 & 19.78 & 21.81 & 65.08 & 2.13 & 70.13 & 8.73 & 19.78 & 19.36 & 65.87 & 1.99 \\
\hline DIR 11 & 63.10 & 12.93 & -12.74 & 18.16 & 315.43 & 1.76 & 70.51 & 10.39 & -11.32 & 15.36 & 312.53 & 1.40 \\
\hline
\end{tabular}

\section{Dyeing of Polyester at 3V}

Dyeing of polyester with DIO 13 showed K/S value of 1.85 and the wash fastness value obtained was moderate at 1.78 (Table-5).

Table-5: Dyeing of polyester at 3V

\begin{tabular}{c|c|c|c|c|c|c|c|c|c|c|c|c}
\hline \multicolumn{1}{c}{ CCM Data before Washing } & \multicolumn{6}{c|}{ CCM Data after Washing } \\
\hline Dye & $\mathrm{L}^{*}$ & $\mathrm{a}^{*}$ & $\mathrm{~b}^{*}$ & $\mathrm{C}^{*}$ & $\mathrm{H}$ & $\mathrm{K} / \mathrm{S}$ & $\mathrm{L}^{*}$ & $\mathrm{a}^{*}$ & $\mathrm{~b}^{*}$ & $\mathrm{C}^{*}$ & $\mathrm{H}$ & $\mathrm{K} / \mathrm{S}$ \\
\hline DIO 3 & 69.63 & 7.79 & 16.33 & 18.09 & 64.50 & 1.77 & 68.63 & 8.31 & 18.35 & 16.15 & 65.62 & 1.60 \\
\hline $\begin{array}{c}\text { DIO } \\
\text { 13 }\end{array}$ & 69.93 & 7.46 & 16.45 & 18.06 & 65.61 & 1.85 & 69.43 & 9.02 & 14.15 & 16.78 & 57.50 & 1.78 \\
\hline $\begin{array}{c}\text { DIR } \\
11\end{array}$ & 63.36 & 15.66 & - & 20.60 & 319.48 & 1.75 & 67.74 & 13.94 & - & 18.70 & 318.21 & 1.34 \\
\hline
\end{tabular}

\section{Dyeing of Polyester at 4V}

Dyeing of polyester fabric with DIO 13 showed K/S value of 1.82 , and 1.75 for the wash fast tested fabric( Table-6).

Table-6: Dyeing of Polyester at 4V

\begin{tabular}{c|c|c|c|c|c|c|c|c|c|c|c|c}
\hline \multicolumn{7}{c|}{ CCM Data before Washing } & \multicolumn{5}{c}{ CCM Data after Washing } \\
\hline Dye & $\mathrm{L}^{*}$ & $\mathrm{a}^{*}$ & $\mathrm{~b}^{*}$ & $\mathrm{C}^{*}$ & $\mathrm{H}$ & $\mathrm{K} / \mathrm{S}$ & $\mathrm{L}^{*}$ & $\mathrm{a}^{*}$ & $\mathrm{~b}^{*}$ & $\mathrm{C}^{*}$ & $\mathrm{H}$ & $\mathrm{K} / \mathrm{S}$ \\
\hline DIO 3 & 69.14 & 8.50 & 16.94 & 18.95 & 63.36 & 1.61 & 70.51 & 6.73 & 13.55 & 15.13 & 63.60 & 1.55 \\
\hline $\begin{array}{c}\text { DIO } \\
\text { 13 }\end{array}$ & 66.82 & 11.88 & 26.61 & 29.14 & 65.95 & 1.82 & 69.64 & 7.37 & 17.19 & 18.71 & 66.80 & 1.75 \\
\hline $\begin{array}{c}\text { DIR } \\
11\end{array}$ & 64.78 & 15.73 & -8.80 & 18.20 & 330.76 & 1.70 & 65.57 & 13.68 & -7.00 & 15.37 & 332.91 & 1.30 \\
\hline
\end{tabular}

It was found that the hue angle for DIO 3 and DIO 13 falls between 36.88 and 89.73 as "a" and "b" are positive. The hue angle for DIR 11 falls between 315.39 and 359.05 as "a" is positive and "b" is negative (Table-7).

Table-7: Comparison of K/S Results in the Electrochemical Dyeing Method

$$
\text { Colour strength }(\mathrm{K} / \mathrm{S})
$$

\begin{tabular}{c|c|c|c|c|c|c}
\multirow{2}{*}{ Dye } & \multicolumn{3}{c|}{ Dyed Sample before Washing } & \multicolumn{3}{c}{ Dyed Sample after Washing } \\
\cline { 2 - 7 } & $2 \mathrm{~V}$ & $3 \mathrm{~V}$ & $4 \mathrm{~V}$ & $2 \mathrm{~V}$ & $3 \mathrm{~V}$ & $4 \mathrm{~V}$ \\
\hline $\begin{array}{c}\text { DISPERSE ORANGE 3 } \\
\text { (DIO3) }\end{array}$ & 1.87 & 1.77 & 1.61 & 1.72 & 1.60 & 1.55 \\
\hline $\begin{array}{c}\text { DISPERSE ORANGE 13 } \\
\text { (DIO13) }\end{array}$ & 2.13 & 1.85 & 1.82 & 1.99 & 1.78 & 1.75 \\
\hline $\begin{array}{c}\text { DISPERSE RED 11 } \\
\text { (DIR11) }\end{array}$ & 1.76 & 1.75 & 1.70 & 1.40 & 1.34 & 1.30 \\
\hline
\end{tabular}


It was inferred (Table-6) that Disperse orange 13 produced better dyeability than other dyes. It was observed that the dyeability decreases as the voltage increases due to the deformation in the dye molecule. Better results were obtained for the disperse dyes with a large gap in peak potential between the peaks (anodic and cathodic). It was observed that the dyeability was found to be better in electrochemical than conventional dyeing 8 .

\section{CONCLUSION}

Dyeing of polyester fabric using disperse dyes by the electrochemical method has greater significance by saving energy when dyed at a lower potential with a better uptake, Since no heat is applied in this technique, it can be considered a greener one.

\section{REFERENCES}

1. A. M. Bond, F. Marken, E. Hill, R. G. Compton, H. Hugel, J. Chem. Soc., Perkin Trans 28, 1735 (1997).

2. S. Komorsky-Lovric, J. Electroanal. Chem, 482, 222 (2000).

3. W. Schrott, ITB International Textile Bulletin May 2000.

4. T. Bechtold US Patent No. US 5244549; 1993-09-14.

5. A. Roessler, X. Jin, Dyes Pigments, 59, 223 (2003).

6. A. Roessler, D. Crettenand, O. Dossenbach, P. Rys, J. Appl. Electrochem., 33, 901 (2003).

7. G Mohamed Zakriya, G. Ramakrishnan, T. Palani Rajan, D. Abinaya, J. Indus. Text., 46, 1393(2017), DOI: $10.1177 / 1528083715624258$

8. K. Kalapriya, H. Gurumallesh Prabu, S. Nithya, Rasayan. J. Chem., 10, 1330(2017), DOI: 10.7324/RJC.2017.1041923

[RJC-5004/2013] 